\title{
Simple parametrization of cross-sections for nuclear transport studies up to the GeV range
}

\author{
J. Cugnon ${ }^{\text {a. * }}$, D. L'Hôte ${ }^{\text {b }}$, J. Vandermeulen ${ }^{a}$ \\ ${ }^{a}$ Université de Liège. Institut de Physique B5, Sart Tilman, B-4000 Liège 1, Belgium \\ ${ }^{b}$ SPEC. CEX - Saclay, F-91191 Gif-sur-Yvette Cedex. France
}

Received 8 September 1995; revised form received 10 November 1995

\begin{abstract}
Simple and accurate parametrizations of nucleon-nucleon and pion-nucleon cross-sections used in nuclear transport codes up to the $1 \mathrm{GeV}$ per nucleon range are presented.
\end{abstract}

\section{Introduction}

The study of heavy ion collisions up to the $\mathrm{GeV}$ range by means of simulations of nuclear transport thcory has undergone a tremendeous impetus in the last ten years (see e.g. Refs. [1-3]) with the successive development of especially the intranuclear cascade [4-6], the BUU model [7-10], the Landau-Vlassov model [11-13], and the QMD model $[14,15]$. It is an important feature for the study of transport of particles inside matter bombarded by heavy ion beams. The same methods have since been extended to hadron-nucleus collisions [16] and antiproton-nucleus interactions $[17,18]$. It is not yet clear whether these theories are in a satisfactory shape, nor that the simulations are really solving the nuclear transport equations correctly [19]. Just to cite an important pending problem, recent investigations [20] have shown, in simple models, that the retardation effects, due to quantum mechanics, in the so-called collision terms are important, although, as far as we know, these effects have not been implemented in a full 3D transport calculation.

An important input of transport theories are the elementary elastic and inelastic nucleon-nucleon collision crosssections (for the inelastic ones, we will limit ourselves here to pion production, which is overwhelmingly dominant in the considered energy range). Most of the existing codes use, at least partly, a parametrization of these experimental cross-sections, which has been devised in parallel with the development of the Liège intranuclear cascade code [5]. Although a large part, but not the whole, of the

\footnotetext{
* Corresponding author. Tel. +3241663601, fax +324166 23 55, e-mail u2183al@vml.ulg.ac.be.
}

parametrization can be found in the literature at various places $[6,17,21,22]$, we think it is useful to present a comprehensive account of it. Furthermore, we also want to show the accuracy of this parametrization. As it will be shown, the latter often provides a compromise between a faithful reproduction of the data, which could only be obtained in principle as lengthy tables, and simple analytical forms which allow fast reconstruction of the cross-sections. It should be borne in mind that it is not really necessary to reach a perfect description of the cross-sections in calculations which are still blurred by large theoretical (and sometimes also statistical) uncertainties. However, it is nonetheless important to retain the main features of the cross-sections. This is the point of view which prevailed in constructing the parametrization that we are going to present. In addition, in some occasions, we will extent the parametrization outside the momentum ranges for which it was first developed.

\section{Parametrization of nucleon-nucleon cross-sections}

\subsection{Total cross-sections}

For the pp total cross-section, the following parametrization has been proposed [6]

$$
\begin{aligned}
\sigma= & 23.5+1000\left(p_{\mathrm{lab}}-0.7\right)^{4}, \quad p_{\mathrm{lab}}<0.8, \\
= & 23.5+\frac{24.6}{1+\exp \left(-\frac{p_{\mathrm{lab}}-1.2}{0.10}\right)}, \\
& 0.8<p_{\mathrm{lab}}<1.5, \\
= & 41+60\left(p_{\mathrm{lab}}-0.9\right) \exp \left(-1.2 p_{\mathrm{lab}}\right), \\
& 1.5<p_{\mathrm{lab}}<5
\end{aligned}
$$




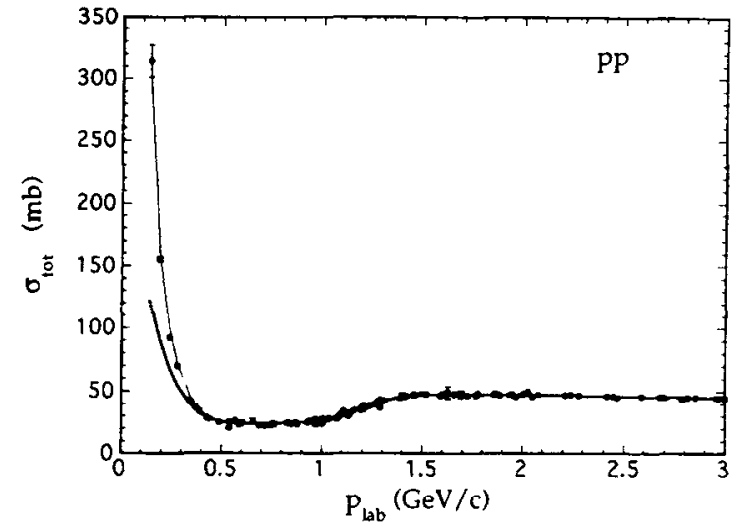

Fig. 1. Comparison of the "world data" [31] for pp cross-section (for $p_{\text {lab }} \leq 0.3 \mathrm{GeV} / c$, only a few points are indicated) and parametrization Eq. (1) indicated by the heavy line. The improved form Eq. (2) for $p_{\text {lab }}<0.4 \mathrm{GeV} / c$ is given by the thin line.

In this equation (and throughout this paper), the cross-section is expressed in mb and the incident lab momentum in $\mathrm{GeV} / c$. A parametrization in term of the $\mathrm{cm}$ energy can be readily obtained by using the well known kinematical formula. As shown in Fig. 1, Eq. (1) yields a good fit up to $5 \mathrm{GeV} / c$ and down to $p_{\mathrm{lab}} \approx 0.3 \mathrm{GeV} / c$, which corresponds roughly to a proton-proton $\mathrm{cm}$ energy of $25 \mathrm{MeV}$. For many purposes, this is largely sufficient as the soft nucleon-nucleon collisions are cut by the Pauli principle in the early phase of the heavy ion collision process. Furthermore, these soft collisions do not contribute very much to the energy-momentum flow. They rather merely exchange nucleons in this flow. In any case, an improved fit can be obtained by using

$\sigma=34\left(\frac{p_{\text {lab }}}{0.4}\right)^{-2.104}, p_{\text {lab }}<0.4$,

which is valid down to $p_{\mathrm{lab}}=0.1 \mathrm{GeV} / c$, i.e. $2.5 \mathrm{MeV}$ proton-proton $\mathrm{cm}$ energy.

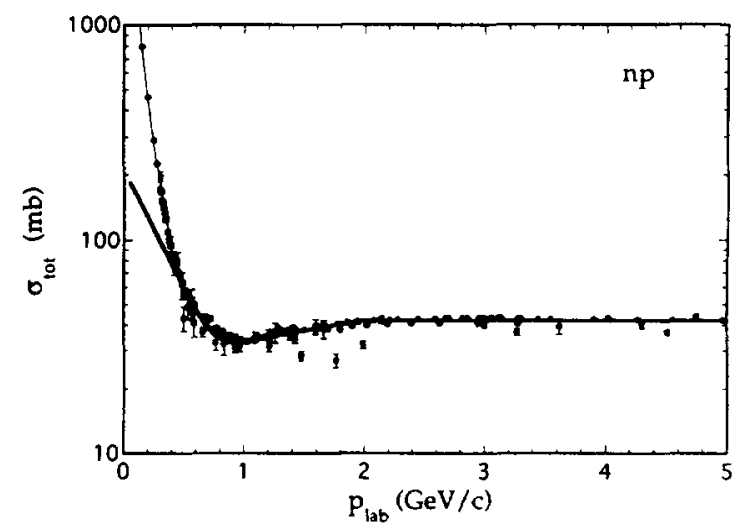

Fig. 2. Comparison of the "world data" [31] for np cross-section and parametrizations Eq. (3) indicated by the heavy line (for $p_{\text {lab }} \leqslant 0.3 \mathrm{GeV} / c$, only a few points are given). The improved form Eq. (4) for $p_{\text {lab }} \leq 0.4 \mathrm{GeV} / c$ is given by the thin line.
In Refs. [17,22], the following parametrization of the total np cross-section has been proposed

$$
\begin{aligned}
\sigma & =33+196\left|p_{\text {lab }}-0.95\right|^{2.5}, & & p_{\text {lab }}<1, \\
& =24.2+8.9 p_{\text {lab }}, & & 1<p_{\text {lab }}<2, \\
& =42, & & 2<p_{\text {lab }} .
\end{aligned}
$$

This parametrization yields a good fit up to $5 \mathrm{GeV} / c$ and down to $0.4 \mathrm{GeV} / \mathrm{C}$ (see Fig. 2). The same remarks apply here as in the pp case. Nevertheless, below $0.4 \mathrm{GeV} / c$, an excellent fit is provided by

$\sigma=6.3555 p_{\mathrm{lab}}^{-3.2481} \exp \left(-0.377\left(\ln p_{\mathrm{lab}}\right)^{2}\right)$,

down to $0.05 \mathrm{GeV} / c$. We note in passing that Eqs. (2) and (4) do not respect the effective range expansions, which, when limited to the scattering length and the effective range, are correct at even smaller incident lab momentum. This is also true for the Coulomb scattering in the pp system. In fact, Eq. (4) is diverging at $p_{\text {lab }}=0$, while the true cross-section is finite. For the pp case, parametrization Eq. (1) on the other hand is diverging at $p_{\mathrm{lab}}=0$, but does not include the Coulomb divergence. These divergences do not however lead to a vanishing mean free path in practical situations, as they are (over) compensated by a vanishingly small phase space factor handling the Pauli blocking. They also lead to a conceptual problem. Interactions are also handled by the mean field introduced in most of transport models. It is natural to consider that the soft collisions (as in particular at very small $p_{\mathrm{lab}}$ ) are mainly contributing to the mean field, whereas hard collisions are entering the collision term only. As far as we know, a satisfactory derivation of a transport theory embodying these considerations is still missing. However, because of the Pauli blocking (as explained above), the soft collisions are expected to be of minor importance.

The parametrizations Eqs. (1)-(4) are not very different from the parametrizations used by the old VEGAS code [23]. They represent however a better statistical average, as they are based on more numerous data. Moreover, they are better at $p_{\text {lab }} \geq 1.5 \mathrm{GeV} / c$.

\subsection{Elastic cross-sections}

For the pp system, the elastic cross-section is practically equal to the total cross-section for $p_{\text {lab }} \leq 1 \mathrm{GeV} / c$. Above this value, the measurements are rather scarce and of a much poorer quality than for the total cross-section (see Fig. 3). In Ref. [6], the following parametrization is used

$$
\begin{array}{rlrl}
\sigma & =23.5+1000\left(p_{\mathrm{lab}}-0.7\right)^{4}, & & p_{\mathrm{lab}}<0.8, \\
& =\frac{1250}{p_{\mathrm{lab}}+50}-4\left(p_{\mathrm{lab}}-1.3\right)^{2}, & & 0.8<p_{\mathrm{lab}}<2, \\
& =\frac{77}{p_{\mathrm{lab}}+1.5}, & 2<p_{\mathrm{lab}} .
\end{array}
$$

It is shown in Fig. 3. 


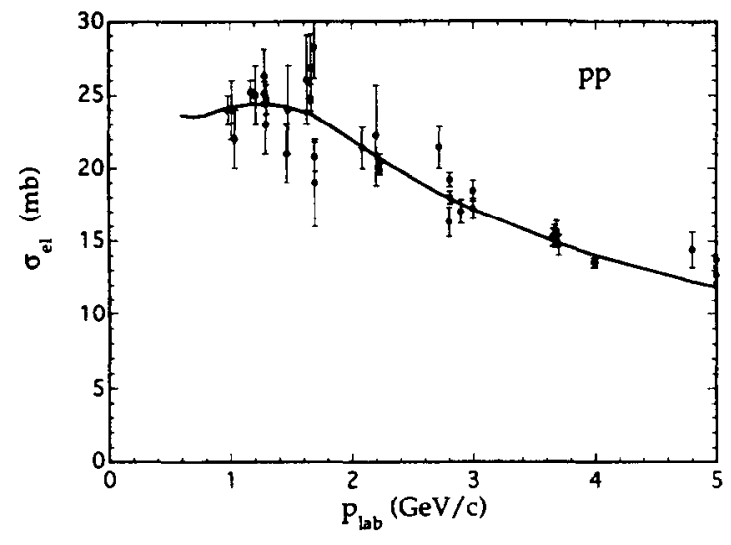

Fig. 3. Comparison of the "world" data [31] for elastic pp cross-section (above the first inelastic threshold) and parametrization Eq. (5).

The elastic data for the $n p$ are even more scarce. A tentative parametrization has been proposed in Ref. [22]:

$$
\begin{aligned}
\sigma & =33+196\left|p_{\text {lab }}-0.95\right|^{2.5}, & & p_{\text {lab }}<0.8, \\
& =\frac{31}{\sqrt{p_{\text {lab }}}}, & & 0.8<p_{\text {lab }}<2, \\
& =\frac{77}{p_{\text {lab }}+1.5}, & & 2<p_{\text {lab }} .
\end{aligned}
$$

It has been built to give a satisfactory description in the 1-5 GeV/c range, with the constraint that the elastic cross-section should be equal to the total cross-section for $p_{\text {lab }} \leq 0.8 \mathrm{GeV} / c$ (see Fig. 4).

\subsection{Differential elastic cross-sections}

We want here to give simple parametrization of the shape of the differential cross-section, contrasting with the

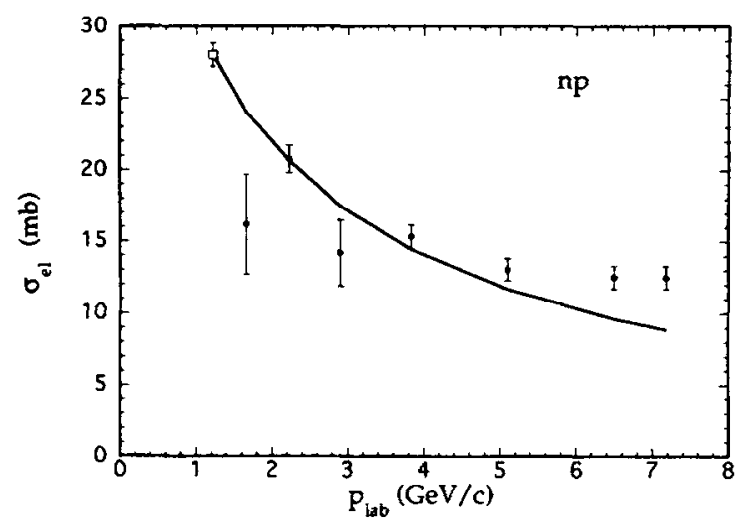

Fig. 4. Comparison of the existing data [31] for elastic np crosssection (above the first inelastic threshold) and parametrization Eq. (6).

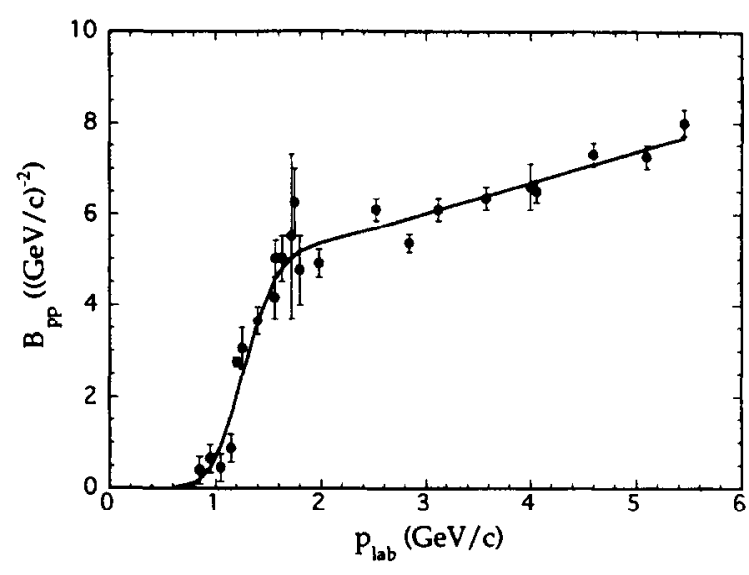

Fig. 5. Comparison of the data $[24,26]$ for the slope parameter $B_{\mathrm{pp}}$ in the pp elastic scattering and parametrization Eq. (8).

description of Ref. [23], which gives it under the form of tables at a few angles and energies.

The differential pp elastic cross-sections can very well be parametrized by the simple form

$$
\frac{\mathrm{d} \sigma}{\mathrm{d} t} \propto \mathrm{e}^{B_{\mathrm{pp}} t},
$$

where $t$ is the usual Mandelstam variable and where $B_{\mathrm{pp}}$ is a function of the cm energy, or equivalently of $p_{\mathrm{lab}}$ :

$$
\begin{array}{rlrl}
B_{\mathrm{pp}} & =\frac{5.5 p_{\mathrm{lab}}^{8}}{7.7+p_{\mathrm{lab}}^{8}}, & & p_{\mathrm{lab}}<2, \\
& =5.334+0.67\left(p_{\mathrm{lab}}-2\right), & 2<p_{\mathrm{lab}} .
\end{array}
$$

It is shown in Fig. 5. Of course, the true cross-section is the symmetrized form of Eq. (7), but for generating the final state in the proton-proton centre of mass, Eq. (7) is sufficient, since if a proton is emitted at an angle $\theta_{\mathrm{cm}}$, another proton is emitted at the angle $\pi-\theta_{\mathrm{cm}}$. Eq. (8), below $2 \mathrm{GeV} / c$, is a slight modification of the shape given in Ref. [24], where it is shown to be quite accurate.

The differential np elastic cross-section is a little bit more complicated. In Ref. [25], the following form has been used:

$$
\frac{\mathrm{d} \sigma}{\mathrm{d} t} \alpha \mathrm{e}^{B_{\mathrm{np}} t}+a \mathrm{e}^{B_{\mathrm{np}} u},
$$

where $t$ and $u$ are the Mandelstam variables. The quantity $B_{\mathrm{np}}$ is given by

$$
\begin{aligned}
B_{\mathrm{np}} & =0, & & p_{\mathrm{lab}}<0.225, \\
& =16.53\left(p_{\mathrm{lab}}-0.225\right), & & 0.225<p_{\mathrm{lab}}<0.6, \\
& =-1.63 p_{\mathrm{lab}}+7.16, & & 0.6<p_{\mathrm{lab}}<1.6, \\
& =B_{\mathrm{pp}}, & & 1.6<p_{\mathrm{lab}} .
\end{aligned}
$$




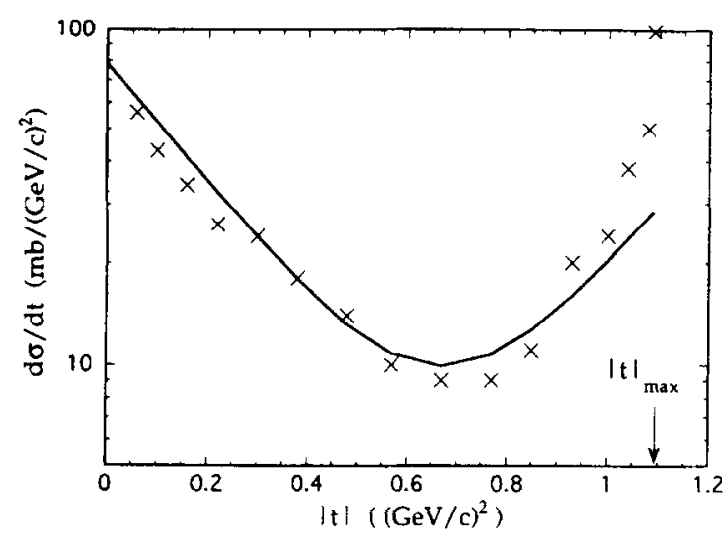

Fig. 6. Comparison of the differential np cross-section (crosses) at $p_{\text {lab }}=1.196 \mathrm{GeV} / c$ and parametrization Eqs. (9)-(11) (full curve). The arrow indicates the value of $t$ corresponding to backward scattering in the $\mathrm{cm}$.

The quantity $a$ is given by

$$
\begin{aligned}
a & =1, & & p_{\text {lab }}<0.8, \\
& =\frac{0.64}{p_{\text {lab }}^{2}}, & & 0.8<p_{\text {lab }} .
\end{aligned}
$$

When $a$ is equal to 1 , the angular distribution is symmetric in the $\mathrm{cm}$ frame and for smaller and smaller values of $a$, the angular distribution gets more and more forward peaked.

The accuracy of parametrization Eq. (10) is really good for $p_{\text {lab }} \leq 0.3 \mathrm{GeV} / c$, where the cross-section is isotropic due to the s-wave dominance and above $p_{\text {lab }} \approx 1.5 \mathrm{GeV} / c$ where the first term of Eq. (9) dominates and where the np cross-section is strongly ressembling the pp cross-section. In the $0.3-1.5 \mathrm{GeV} / c$ range, Eq. (9) is again a compromise between accuracy and convenience. Indeed, to generate the final state, it is sufficient first to determine (by comparing a random number to $a /(1+a)$ ) whether the $\mathrm{e}^{B_{\mathrm{np}} t}$ or $\mathrm{e}^{B_{\mathrm{pp}} u}$ form is chosen and to determine the value of $t$ and $u$ by using an exponential random number. In practice, it is even simpler: it is sufficient to determine the final state by $\mathrm{e}^{B_{\mathrm{np}} t}$ and a fraction $a /(1+a)$ of the time, to exchange neutron and proton. The accuracy of Eq. (9) in the $0.6-1.5 \mathrm{GeV} / c$ range is illustrated in a typical case in Fig. 6. Parametrization Eq. (9) neglects the rapid increase close to $|t|_{\max }$, i.e. to $\theta_{\mathrm{cm}}=180^{\circ}$, which corresponds to the pion exchange and which is limited to a narrow range of angles and, therefore, a small part of the integrated crosssection.

The $p_{\mathrm{lab}}$ dependence of $B_{\mathrm{np}}$ and $a$ is given in Fig. 7.

\subsection{Inelastic cross-sections}

The inelastic cross-sections are simply the differences between the total and elastic cross-sections. Below, say 3.5 $\mathrm{GeV} / c$, the inelasticity is dominated by the one pion production channel. Therc arc many indications that, in this momentum range, the pion production largely proceeds through the formation of a $\Delta$ resonance (this cannot be true for the whole inelastic cross-section, as the $\mathrm{np}$ and pp inelastic cross-sections are not exactly in the ratio $\frac{1}{2}$, that is required by the pure isobar model). Nevertheless, a picture based on the $\Delta$ production has been suggested and yields reasonable results. In Ref. [21], it is proposed to adopt the following scenario: a variable mass $\Delta$ is produced, which can decay after some time. The distribution of the $\Delta$ mass $M$ is given by

$$
\begin{aligned}
f(M) \propto & \frac{1}{\left(M-M_{0}\right)^{2}+\frac{\Gamma_{0}^{2}}{4}} \theta\left(M-M_{\mathrm{n}}-m_{\pi}\right) \\
& \times \theta\left(\sqrt{s}-M_{\mathrm{n}}-M\right),
\end{aligned}
$$

where $M_{0}$ and $\Gamma_{0}$ are the fundamental parameters of the $\Delta$ resonance, namely $M_{0}=1.232 \mathrm{GeV}, \Gamma_{0}=0.115 \mathrm{GeV}$, where $M_{\mathrm{n}}$ is the nucleon mass, $m_{\bar{\pi}}$ the pion mass and $\sqrt{s}$ is the $\mathrm{cm}$ energy. In practice, it is sufficient to generate a $\Delta$ mass according to a Lorentzian distribution repeatedly, until the two conditions expressed by the $\theta$ functions in Eq. (13) are satisfied.

The angular distribution of the $\Delta$ production reaction can be described as follows [6,21]:

$\frac{\mathrm{d} \sigma}{\mathrm{d} t}(\mathrm{NN} \rightarrow \mathrm{N} \Delta) \propto \mathrm{e}^{B_{\ln } t}$,

with

$B_{\text {in }}=B_{\mathrm{pp}}$

at the same $\mathrm{cm}$ energy, where $t$ is the square of the four-momentum transfer from the incident nucleon to the $\Delta$ resonance. This is more or less supported by the experimental data [26,27] (see Fig. 8).

Let us remind that, in this scenario [6], the $\Delta$ resonance is assumed to decay isotropically in its rest frame, which

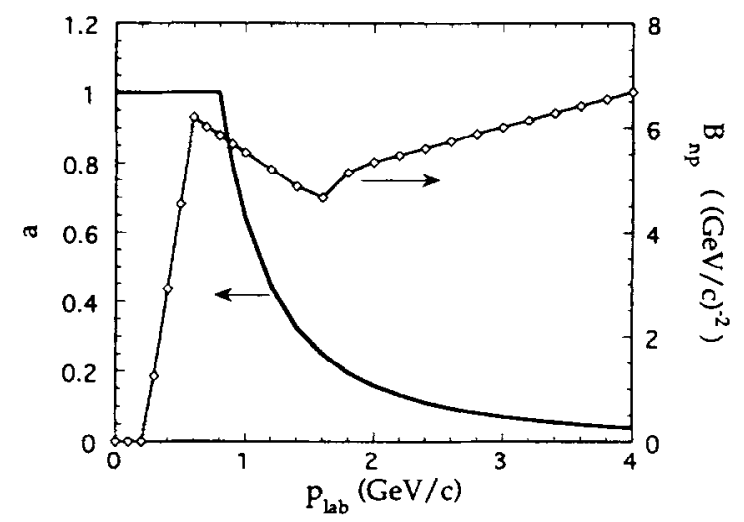

Fig. 7. Incident momentum dependence of the parameters $a$ (Eq. (11)), left scale) and $B_{\mathrm{np}}$ (Eq. (10)), right scale). 


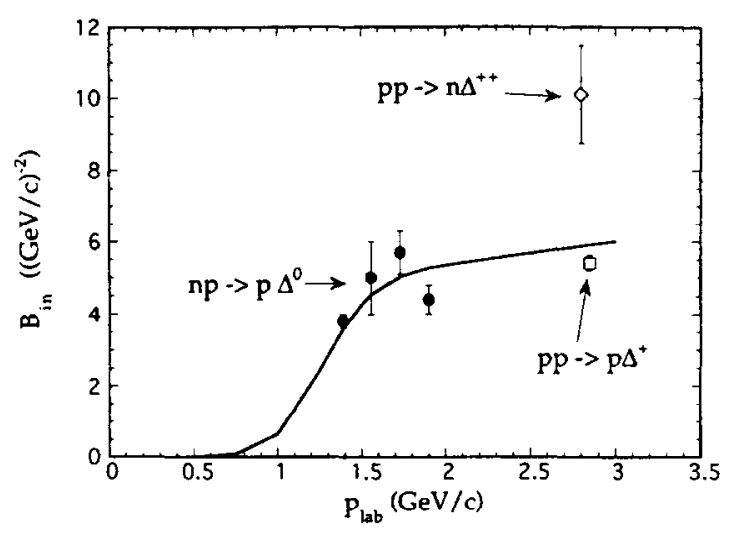

Fig. 8. Comparison of the existing data for the slope parameter $B_{\text {in }}$ and the parametrization given by Eq. (14).

seems to introduce some error in the pion angular distribution in the nucleon-nucleon frame at low energy. Improving on this point would require a model for the distribution of the $\Delta$ spin in the $\Delta$ production and to keep track of the direction of this spin. Finally, the various branching ratios, both for $\Delta$ production and decay, are assumed to be given by the isobar model

$$
\begin{aligned}
& \frac{\sigma\left(\mathrm{pp} \rightarrow \mathrm{p} \Delta^{+}\right)}{\sigma\left(\mathrm{pp} \rightarrow \mathrm{n} \Delta^{++}\right)}=\frac{\sigma\left(\mathrm{nn} \rightarrow \mathrm{n} \Delta^{0}\right)}{\sigma\left(\mathrm{nn} \rightarrow \mathrm{p} \Delta^{-}\right)}=\frac{1}{3}, \\
& \frac{\sigma\left(\mathrm{np} \rightarrow \mathrm{n} \Delta^{+}\right)}{\sigma\left(\mathrm{np} \rightarrow \mathrm{p} \Delta^{0}\right)}=1, \\
& \frac{\Gamma\left(\Delta^{+} \rightarrow \pi^{+} \mathrm{n}\right)}{\Gamma\left(\Delta^{+} \rightarrow \pi^{0} \mathrm{p}\right)}=\frac{\Gamma\left(\Delta^{0} \rightarrow \pi^{-} \mathrm{p}\right)}{\Gamma\left(\Delta^{0} \rightarrow \pi^{0} \mathrm{n}\right)}=\frac{1}{2}
\end{aligned}
$$

\section{Parametrization of pion-nucleon cross-sections}

In the energy range considered here, the pion-nucleon interaction is largely dominated by the $(3,3)$ resonance. It is then legitimate to adopt the pure isobar model. The following parametrization has been proposed in Ref. [21] for the $\Delta^{++}$formation

$$
\sigma\left(\pi^{+} \mathrm{p} \rightarrow \Delta^{++}\right)=\frac{326.5}{1+4\left(\frac{\sqrt{s}-1.215}{0.110}\right)^{2}} \frac{q^{3}}{q^{3}+(0.18)^{3}},
$$

where $q$ is the $\mathrm{cm}$ momentum

$$
\begin{aligned}
q & =\left[\frac{\left(s-\left(m_{\pi}+m_{\mathrm{p}}\right)^{2}\right)\left(s-\left(m_{\pi}-m_{\mathrm{p}}\right)^{2}\right)}{4 s}\right]^{1 / 2} \\
& =\frac{m_{\mathrm{p}}}{\sqrt{s}} p_{\mathrm{lab}} .
\end{aligned}
$$

The departure from a pure Lorentzian form (as in Ref. [23]) has been adopted to account for the momentum dependence of the $\Delta$ partial width in the $\pi p$ channel [28], as dictated by the experimental data, which clearly show an asymmetry of the $\Delta$ resonance in the $\pi p$ elastic cross-section. As can be seen from Fig. 9, formula Eq. (16) provides an accurate parametrization of the total $\pi^{+} p$ cross-section for $p_{\mathrm{lab}} \leq 0.7 \mathrm{GeV} / c$, i.e. for pions of incident energies less than $\sim 0.6 \mathrm{GeV}$. This is largely sufficient for the domain we consider here. The cross-sections for other isospin channels are easily obtained in the isobar model. One has

$$
\begin{aligned}
\sigma\left(\pi^{+} \mathrm{p} \rightarrow \Delta^{++}\right) & =3 \sigma\left(\pi^{+} \mathrm{n} \rightarrow \Delta^{+}\right)=\frac{3}{2} \sigma\left(\pi^{0} \mathrm{p} \rightarrow \Delta^{+}\right) \\
& =\frac{3}{2} \sigma\left(\pi^{0} \mathrm{n} \rightarrow \Delta^{0}\right)=3 \sigma\left(\pi^{-} \mathrm{p} \rightarrow \Delta^{0}\right) \\
& =\sigma\left(\pi^{-} \mathrm{n} \rightarrow \Delta^{-}\right) .
\end{aligned}
$$

Let us notice that this relation is almost perfectly verificd for $p_{\mathrm{lab}} \leq 0.5 \mathrm{GeV} / c$, by the two accurately measured cross-sections, namely $\pi^{+} p \rightarrow \Delta^{++}$and $\pi^{-} p \rightarrow \Delta^{0}$. Above $0.5 \mathrm{GeV} / c$, deviations from the pure isobar model start to show up in the last reaction.

Above $p_{\text {lab }} \approx 0.7 \mathrm{GeV} / c$, the $\pi-\mathrm{N}$ dynamics becomes complicated. From $0.7 \mathrm{GeV} / c$ to $\approx 1.2 \mathrm{GeV} / c$, the $\pi^{+} \mathrm{p}$ and $\pi^{-} p$ systems are dominated by different broad resonances. However, as indicated in Ref. [17], the isospin averaged $\pi \mathrm{N}$ cross-section is rather smoothly energy-dependent. Therefore, we will limit ourselves to propose parametrizations for isospin averaged cross-sections. For the $\pi \mathrm{N}$ elastic cross-section, it is suggested in Refs. $[17,29]$ to take $\sigma_{\mathrm{el}}(\pi \mathrm{N}) \approx 30 \mathrm{mb}$ in the $0.6-2 \mathrm{GeV} / \mathrm{c}$ range. In the $0.5-1.5 \mathrm{GeV} / c$ range, the inelastic $\pi \mathrm{N}$ cross-section is largely dominated by the $\pi$ production $(\pi \mathrm{N} \rightarrow \pi \pi \mathrm{N})$. For the latter, a good description of the isospin average cross-section is given by

$\sigma(\pi \mathrm{N} \rightarrow \pi \pi \mathrm{N})=74\left(p_{\mathrm{lab}}-0.555\right)^{2} p_{\text {lab }}^{-4.04}$,

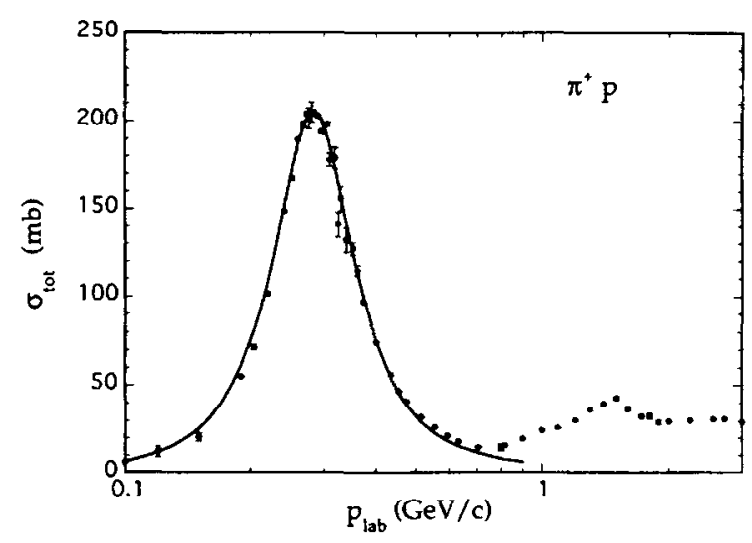

Fig. 9. Comparison of the "world" data [31] for the total $\pi^{+} p$ cross-section (actually, only selected representative points are given), with parametrization Eq. (16). 


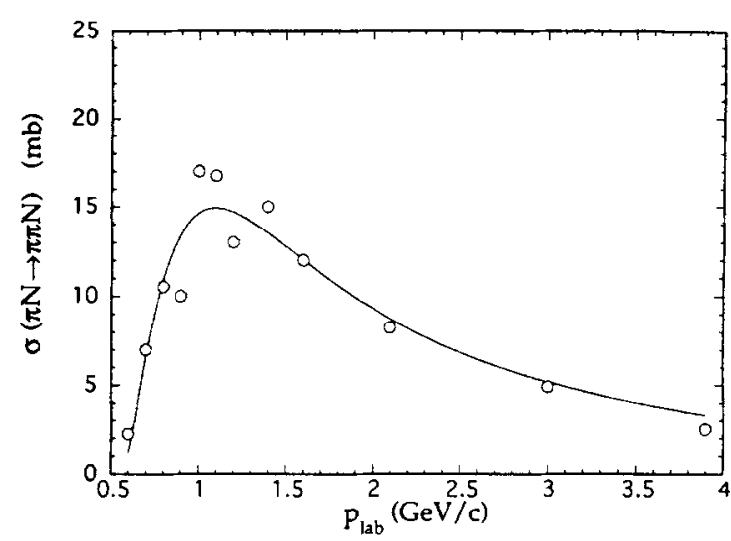

Fig. 10. The dots give the isospin average $\pi \mathrm{N} \rightarrow \pi \pi \mathrm{N}$ cross-section, obtained by summing all measured cross-sections for specific charged final states. The line corresponds to Eq. (19).

as can be seen from Fig. 10. In Refs. [17,29], the elastic scattering above $0.5 \mathrm{GeV} / c$ is still assumed for the sake of simplicity to proceed through the $\Delta$-resonance, which is certainly not corresponding to the physical reality, and the pion production is assumed to populate isotropically the available phase space, which is also an approximation. These simple choices seem however to produce reasonable results [17].

\section{Conclusion}

In this paper, we summarized the parametrizations of the nucleon-nucleon and pion-nucleon cross-sections proposed by our group, of which a partial account appeared in the literature. We exhibited their good accuracy in the momentum ranges relevant for the transport theory calculations for heavy ion collisions up to $\sim 2 \mathrm{GeV} / \mathrm{u}$ incident energy, for proton-nucleus collisions up to $\sim 2 \mathrm{GeV}$ and antiproton-nucleus annihilations up to $\sim 1 \mathrm{GeV}$. In some occasions, we extended the parametrizations to go somewhat above these limits. However, let us conclude in mentioning that the real limitations for this extension are then coming from the very description of the inelastic collisions, which may lead to several particle final states (directly or in two or three steps) and the increasing variety of produced particles. Some helpful parametrizations in these directions can be found in Ref. [30].

\section{References}

[1] L.P. Csernai and D.D. Strottmann, Int. Rev. Nucl. Phys. 5 (1991) 37.
[2] J. Cugnon, in: Particle Production in Highly Excited Matter, eds. H.H. Gutbrod and J. Rafelski. NATO ASI Series B: vol. 303 (Plenum, New York, 1993) p. 271.

[3] G.F. Bertsch and S. Das Gupta, Phys. Rep. C 160 (1988) 189.

[4] Y. Yariv and Z. Fraenkel, Phys. Rev. C 20 (1979) 2227.

[5] J. Cugnon, Phys. Rev. C 22 (1980) 1885.

[6] J. Cugnon, T. Mizutani and J. Vandermeulen, Nucl. Phys. A 352 (1981) 505.

[7] G.F. Bertsch, H. Kruse and S. Das Gupta, Phys. Rev. C 29 (1984) 673.

[8] J.J. Molitoris, J.B. Hoffer, H. Kruse and H. Stöcker. Phys. Rev. Lett. 53 (1984) 899.

[9] C. Gale, G.F. Bertsch and S. Das Gupta, Phys. Rev. C 35 (1987) 1666.

[10] W. Cassing et al., Phys. Rep. C 188 (1990) 363.

[11] E. Suraud, G. Grégoire and B. Tamain, Prog. Part. Nucl. Phys. 23 (1989) 357.

[12] B. Rémaud, C. Grégoire, F. Sébille and P. Schuck, Nucl. Phys. A 488 (1988) 423c.

[13] P. Schuck, R.N. Hasse, J. Janicke, C. Grégoire, B. Rémaud, F. Sébille and E. Suraud, Prog. Part. Nucl. Phys. 25 (1990) 235.

[14] J. Aichelin, Phys. Rep. C 202 (1991) 233.

[15] J. Aichelin, G. Peilert, A. Bohnet, A. Rosenhauer, H. Stöcker and W. Greiner, Phys. Rev. C 37 (1988) 2451.

[16] J. Cugnon, Nucl. Phys. A 462 (1987) 751.

[17] J. Cugnon, P. Deneye and J. Vandermeulen, Nucl. Phys. A 500 (1989) 701.

[18] Ye.S. Golubeva, A.S. Iljinov and M.V. Mebel, Nuovo Cimento 103(5) (1990) 781.

[19] G. Batko and J. Randrup, Nucl.Phys. A 563 (1993) 97.

[20] R. Malfliet, Nucl. Phys. A 545 (1992) 3c.

[21] J. Cugnon, D. Kinet and J. Vandermeulen, Nucl. Phys. A 379 (1982) 553.

[22] J. Cugnon and M.-C. Lemaire, Nucl. Phys. A 489 (1988) 781.

[23] K. Chen, Z. Fraenkel, G. Friedlander, J.R. Grover, J.M. Miller and Y. Shimamoto, Phys. Rev. 166 (1968) 949.

[24] G.J. Igo, Rev. Mod. Phys. 50 (1978) 523.

[25] J. Cugnon and D. L'Hôte, Nucl. Phys. A 452 (1986) 738.

[26] The Particle Data Group, NN and ND Interactions (above 0.5 $\mathrm{GeV} / \mathrm{c}$ ). A compilation. UCRL-20000 NN (1970).

[27] J.L. Laville, Ph.D. Thesis, University of Caen (1976).

[28] H.M. Pilkuhn, Relativistic Particle Physics (Springer, 1979, New York).

[29] J. Cugnon, Nucl. Phys. A 542 (1992) 559.

[30] P. Deneye, Ph.D. Thesis, University of Liège (1991).

[31] A. Baldini, V. Flaminio, W.G. Moorhead and D.R.O. Morrison, Landolt-Börnstein, New Series, Group I, Vol. 12a (Springer, Berlin, 1988). 